\title{
Germanium Mid-Infrared Photonic Devices
}

\author{
Goran Z. Mashanovich, Colin J. Mitchell, Jordi Soler Penades, Ali Z. Khokhar, Callum G. Littlejohns, \\ Wei Cao, Zhibo Qu, Stevan Stankovic, Frederic Y. Gardes, Taha Ben Masaud, Harold M. H. Chong, \\ Vinita Mittal, Ganapathy Senthil Murugan, James S. Wilkinson, Anna C. Peacock, and \\ Milos Nedeljkovic
}

\begin{abstract}
Germanium based photonic devices can play a significant role in several applications, particularly in the socalled fingerprint wavelength region. Here, we review our recent results on mid-infrared germanium photonic devices that show promising performance in the $2-7.5 \mu \mathrm{m}$ wavelength range.
\end{abstract}

Index Terms-Germanium, silicon photonics, waveguides, couplers, interferometers

\section{INTRODUCTION}

$\mathrm{M}$ ID-INFRARED (MIR) group IV photonic devices and systems show promise in a range of applications such as environmental and biochemical sensing, industrial process control, detection of toxics, point of care diagnostics, astronomy or communications. The so-called fingerprint wavelength region $(6-15 \mu \mathrm{m})$ is particularly useful for sensing as many molecules show strong absorption features at distinct wavelengths in this region [1]. On the other hand, the 2-2.6 $\mu \mathrm{m}$ range is becoming important for telecom/datacom applications [2]. The latter application area can utilize siliconon-insulator (SOI) as advanced design, fabrication and testing techniques of SOI devices have been developed over the years and SOI is the most mature group IV photonics technology available.

The former application area does, however, require new material platforms, because silicon dioxide is lossy beyond $\sim 4$ $\mu \mathrm{m}$ and therefore SOI cannot be used at longer wavelengths [1]. Several alternatives have been investigated: silicon on

Manuscript received July 1, 2016; revised October 16, 2016; accepted October 28, 2016. Date of publication Month date, year; date of current version Month date, year. This work was supported in part by Goran Mashanovich's Royal Society Research Fellowship (UF120053), EPSRC projects MIGRATION (EP/L01162X/1), "Silicon Photonics for Future Systems" (EP/L00044X/1) and CORNERSTONE (EP/L021129/1), and by the European Research Council (ERC) grant 291216 "Wideband Integrated Photonics for Accessible Biomedical Diagnostics."

G. Z. Mashanovich, C. J. Mitchell, J. Soler Penades, A. Z. Khokhar, C. G. Littlejohns, W. Cao, Z. Qu, S. Stankovic, F. Y. Gardes, V. Mittal, G. Senthil Murugan, J. S. Wilkinson, A. C. Peacock, and M. Nedeljkovic are with the Optoelectronics Research Centre, University of Southampton, Southampton, Hampshire, SO17 1BJ, UK (e-mail: g.mashanovich@soton.ac.uk). G. Z. Mashanovich is also with the School of Electrical Engineering, University of Belgrade, 11120 Belgrade, Serbia.

T. Ben Masaud and H. M. H. Chong are with the Electronics and Computer Science department, University of Southampton, Southampton, Hampshire, SO17 1BJ, UK.

Data published in this paper are available from the University of Southampton repository at $10.5258 /$ SOTON/401414 sapphire (SOS) [3-5], silicon on nitride [6], silicon on porous silicon [7], suspended silicon [8-10] and germanium on silicon [11-14].

Germanium-based material platforms are particularly interesting for the realization of MIR photonic devices because Ge has broader MIR transparency range than $\mathrm{Si}$ (2-15 $\mu \mathrm{m})$. It has also higher carrier mobility and larger non linear effects than Si. Germanium core fibers [15] and germanium slabs on zinc sulfide substrates [16] were realized before first reports on Ge-on-Si based MIR planar photonic devices. Chang et.al demonstrated Ge-on-Si (GOS) waveguides with losses of $2.5-3.0 \mathrm{~dB} / \mathrm{cm}$ at $\lambda=5.8 \mu \mathrm{m}$ [11], which was followed by GOS multiplexers $[12,13]$ at $\sim 5.3 \mu \mathrm{m}$, and Mach-Zehnder interferometers and thermo-optic modulators [14] at the same wavelength range.

In this paper, in sections II and III we review our recently reported results on MIR Ge-based photonics devices, and we report new results at longer wavelengths and on amorphous germanium waveguides in sections IV and $\mathrm{V}$, respectively.

\section{TWO MICROMETER THICK GE-ON-SI WAVEGUIDES}

We initiated our investigation of the GOS platform by using $2 \mu \mathrm{m}$ Ge films. Reduced pressure chemical vapor deposition (RPCVD) was used to grown these films on six inch $\mathrm{Si}$ wafers. Threading dislocations appear at the Si-Ge interface due to the lattice constant difference between $\mathrm{Si}$ and $\mathrm{Ge}$, Wet selective defect etch [17] was used to determine a threading dislocation density (TDD) of $2 \times 10^{7}-5 \times 10^{7} \mathrm{~cm}^{-2}$.

\section{A. Fabrication and characterisation}

To fabricate the GOS rib waveguides we used a combination of lithographic patterning and reactive-ion etching (RIE) methods. The ribs were designed to have an etch depth of 1.2 $\mu \mathrm{m}$ and a core width of $2.25 \mu \mathrm{m}$. Coupling into and out of the core region was facilitated via the incorporation of tapered waveguides at each end of the sample, which had a maximum width of $10 \mu \mathrm{m}$. Waveguides of different lengths were incorporated onto the chip by introducing four identical bends, of varying separation, in each waveguide. The top images in Fig. 1 display SEM cross-sectional images of (a) the input facet and (b) the waveguide core, showing that the ribs were well formed in both regions.

To characterize the optical transmission properties of the waveguides, linear loss measurements were performed using various MIR CW sources that covered a wavelength range of 1.9-3.8 $\mu \mathrm{m}$. The cut-back method was applied by comparing 
the transmitted power through waveguides of different lengths. The results of these measurements are summarized in Fig. 1, showing how the losses vary over the entire wavelength range. The high loss value recorded at $1.95 \mu \mathrm{m}$ is expected as it is close to germanium's band edge, but beyond 2 $\mu \mathrm{m}$ the losses flatten out to a consistently low value of $\sim 3$ $\mathrm{dB} / \mathrm{cm}$. This trend indicates that the GOS waveguides should exhibit a broad transmission window across much of the MIR regime provided that there is little overlap with the silicon substrate, which becomes lossy for wavelengths $>8 \mu \mathrm{m}$.

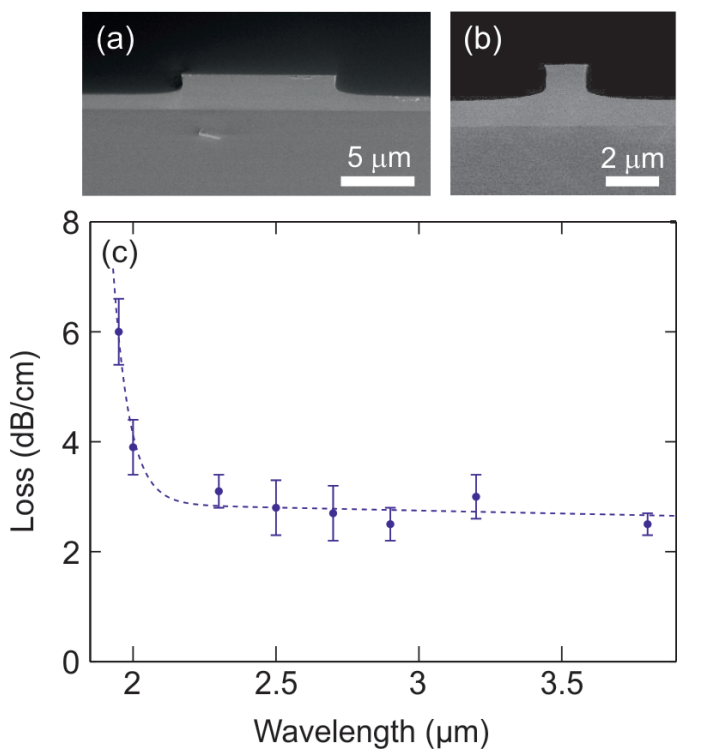

Fig. 1. (a) SEM cross-sectional image of the GOS input taper and (b) the waveguide core. (c) Propagation losses measured over the 1.9-3.8 $\mu \mathrm{m}$ wavelength range [18].

\section{B. Non-linear absorption characterisation}

Although Ge has long been predicted to be of interest for nonlinear applications in the MIR, as of to date it remains a largely untapped area of research. We started to benchmark the nonlinear performance of the GOS waveguides $(1.4 \mathrm{~cm}$ long) by characterizing the two-photon absorption (TPA) strength as a function of wavelength. Several measurements of the TPA parameter $\beta_{\text {TPA }}$ had been previously reported for bulk Ge samples, however these were yet to be performed for $\mathrm{Ge}$ waveguides.

High power transmission experiments were performed in which the saturation of the output power was monitored as a function of increasing input pump power [19]. The pump pulses were delivered from a high power optical parametric oscillator (OPO), with durations of $\sim 250 \mathrm{fs}$ (FWHM) at a 80 $\mathrm{MHz}$ repetition rate [20]. The OPO was tunable over the entire TPA window 1.9-3.7 $\mu \mathrm{m}$. The measurement results for a few selected pump wavelengths are shown in Fig. 2(a). It can be seen that for wavelengths up to $3.4 \mu \mathrm{m}$ the output powers saturate due to the strong nonlinear TPA in this region. On the other hand, at $3.7 \mu \mathrm{m}$ there is a linear trend for transmission which indicates that the TPA parameter has dropped significantly. We fitted the data with the simplified coupled nonlinear Schrödinger equations (NLSEs) described in [20] (solid curves), and obtained the values for $\beta_{\text {TPA }}$ (Fig. 2(b)). This data shows the expected trend of decreasing $\beta_{\text {TPA }}$ for increasing wavelength when moving across the TPA window, and the high recorded values $(1000 \times$ larger than reported for $\mathrm{Si}$ ) were in good agreement with values that had been obtained previously for bulk Ge $[21,22]$.

\section{All-optical modulation}

In many applications large nonlinear absorption coefficients are undesirable. However, owing to the ultrafast nature of TPA, it can be exploited to realize a number of all-optical processing functions such as logic gates, pulse shapers, modulators and switches [23]. For example, using the large $\beta_{\text {TPA }}$ parameter at $\sim 2 \mu \mathrm{m}$ we have demonstrated a high-speed, all-optical cross-absorption modulation (XAM) process. The experiment makes use of a simple pump-probe set-up, where a high power pump is used to induce an absorption dip on a weak probe, which was otherwise too weak to induce TPA. Thus the necessary conditions for this process is that the energy of the two photons, one from the pump and one from the probe, is sufficient to span the bandgap.
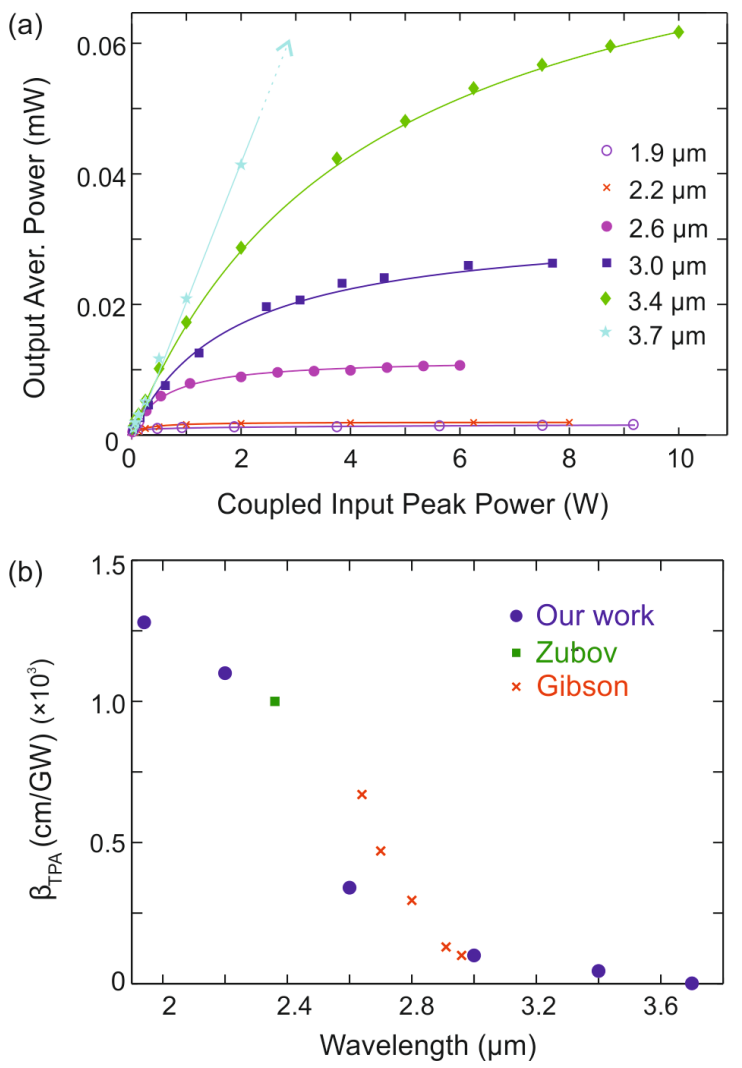

Fig. 2. (a) Nonlinear absorption measurements in the GOS rib waveguides, together with numerical fitted curves from solving the simplified NLSE (solid lines). (b) Calculated TPA parameters as a function of wavelength, together with data points obtained from bulk measurements by Zubov et al. [21] and Gibson et al. [22].

The XAM setup is illustrated in Fig. 3. A mode-locked fiber laser at $1.95 \mu \mathrm{m}$ was combined with a custom built thuliumdoped fiber amplifier (TDFA) to generate the high power pump with a 5 ps (FWHM) pulse duration and $25 \mathrm{MHz}$ repetition rate. The source was split into the weak probe $(\sim 10$ 
$\mathrm{mW}$ coupled peak power) and pump (maximum $10 \mathrm{~W}$ coupled peak power) using a beam-splitter (BS in Fig. 3). The probe was modulated using a chopper that was connected to a lockin amplifier (LA). An optical delay line was also used in the probe arm to introduce a variable time delay between the probe and the pump. The autocorrelator (AC) applied to measure this delay. The two beams are then combined with another BS and coupled into the waveguide with a silica objective lens (O1). The output was collected via a second objective lens $(\mathrm{O} 2)$ and the power measured using a $\mathrm{PbSe}$ detector.

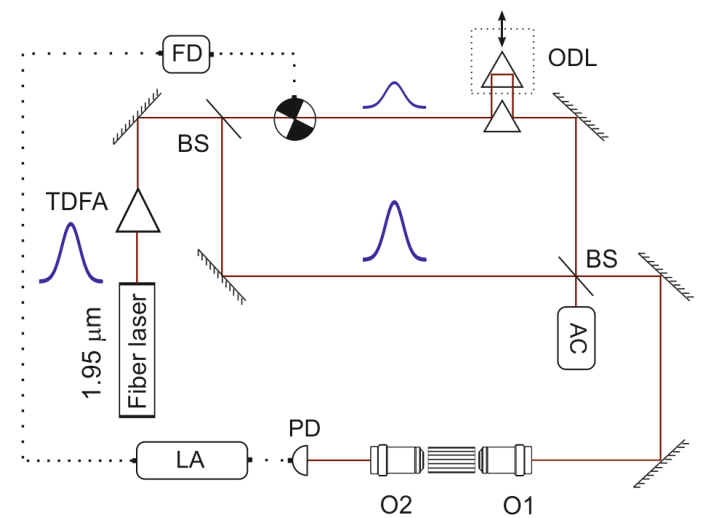

Fig. 3. Experimental setup for all-optical modulation using TPA. Thuliumdoped fibre amplifier (TDFA), optical delay line (ODL), autocorrelator (AC), photodiode (PD), lock-in amplifier (LA), frequency driver (FD), microscope objective lenses (O1 \& O2) and beam-splitter (BS). [20]
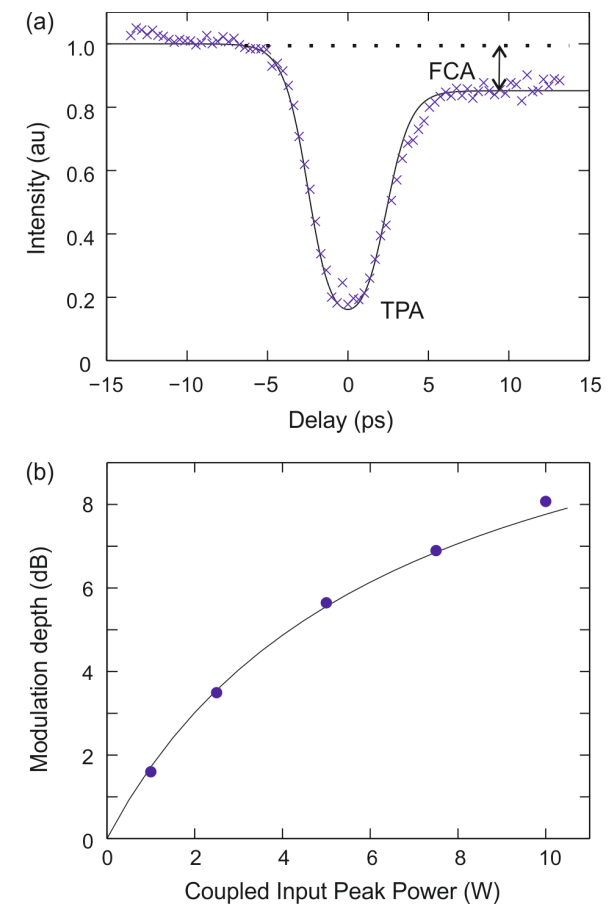

Fig. 4. High speed nonlinear absorption (crosses) of a weak probe, together with a fit obtained via solving the modified NLSEs (solid line). (b) Modulation depth as a function of coupled input pump peak power together with the simulated fit [20].

The absorption of the probe pulse as a function of delay for a coupled pump peak power of $10 \mathrm{~W}$ is given in Fig. 4(a) which clearly shows that the ultrafast absorption due to TPA occurred on the timescale of the pump pulse $\left(\mathrm{T}_{\text {fwhm }} \sim 5 \mathrm{ps}\right)$, before slower recombination mechanisms due to free carrier absorption (FCA) took place. By monitoring the material recovery with a high-speed $10 \mathrm{GHz}$ bandwidth InGaAs photodetector, we estimated the free-carrier lifetime in the GOS waveguides to be $\sim 18 \mathrm{~ns}$, which was faster than what had been measured for $\mathrm{Si}$ waveguides with similar micronsized core dimensions. Fitting the absorption response of the probe beam with the modified NLSE [20], we obtained an excellent agreement (solid line), which verifies the size of the TPA parameter taken from Fig. 2(b). Repeating these measurements for different pump powers produces the trend shown in Fig. 4(b), where the modulation depth increases with power as expected, though with a roll-off associated with the saturating pump.

From Fig. 4(b) it can be seen that the maximum extinction ratio (ER) of $8.1 \mathrm{~dB}$ was achieved. This is the highest reported ER in any of the group IV waveguides to date $[24,25]$. This can be attributed to the very large nonlinear absorption we measured experimentally. As $\beta_{\mathrm{TPA}}$ is large in the 2-3 $\mu \mathrm{m}$ range, this scheme can be extended across this wavelength range to develop high-speed all optical modulators.

\section{THREE MICROMETER THICK GE-ON-SI DEVICES}

After the previous investigation of $2 \mu \mathrm{m}$ GOS waveguides, we moved to the platform with $3 \mu \mathrm{m}$ Ge thick layer on $\mathrm{Si}$ substrate. The main motivation was to reduce the interaction of the optical mode with threading dislocations at the Ge-Si boundary, as well as with the sidewall roughness. In addition, this thicker platform would be more suitable for the fingerprint wavelength region due to larger waveguide dimensions. To enable single mode propagation, rib waveguides were chosen. Their dimensions were determined by modelling using the FMM solver in the Photon Design Fimmwave [26]. The waveguide dimensions for a wavelength of $\lambda=3.8 \mu \mathrm{m}$ were: height $(\mathrm{H})=2.9 \mu \mathrm{m}$, width $(\mathrm{W})=2.7 \mu \mathrm{m}$, etch depth $(\mathrm{D})=$ $1.7 \mu \mathrm{m}$.

Compared to the $2 \mu \mathrm{m}$ structures described in the previous section, we used e-beam lithography and ICP etching rather than optical photolithography and RIE etching. ZEP-520A positive resist, and $\mathrm{SF}_{6}$ and $\mathrm{C}_{4} \mathrm{~F}_{8}$ gases in the ICP [27], were used. Fig. 5 shows a cross section of the fabricated GOS waveguide.

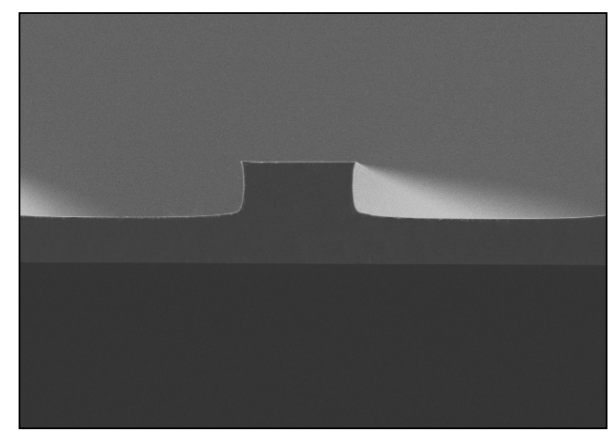

Fig. 5. Cross section of a $3 \mu \mathrm{m}$ GOS waveguide [27] 
Unlike the previous demonstrations of GOS devices [11-14] where samples were cleaved, we used surface grating couplers to couple the light to and from chips, for the first time. Lumerical [28] was used to model the grating couplers. To simplify the fabrication, the grating etch depth was the same as the rib depth $\mathrm{D}=1.7 \mu \mathrm{m}$, whilst the values for the period and duty cycle were $\Lambda=2.0 \mu \mathrm{m}$ and 0.5 , respectively. This first generation of $\mathrm{Ge}$ grating couplers have been further optimized in terms of increased coupling efficiency and reduced reflection, by using inverse taper excitation [29].

A measurements setup described in detail in [7] was used to characterize fabricated devices. The grating couplers were optimized for TE polarization. The propagation loss at $\lambda=3.8$ $\mu \mathrm{m}$ was determined by the cut-back method and a value of $0.58 \pm 0.12 \mathrm{~dB} / \mathrm{cm}$ was measured (Fig. 6). This value is significantly lower than any other previously reported results for the GOS platform [11-14]. We attribute this reduced loss to lower mode interaction with both the Ge-Si interface region and the etched waveguide sidewalls.

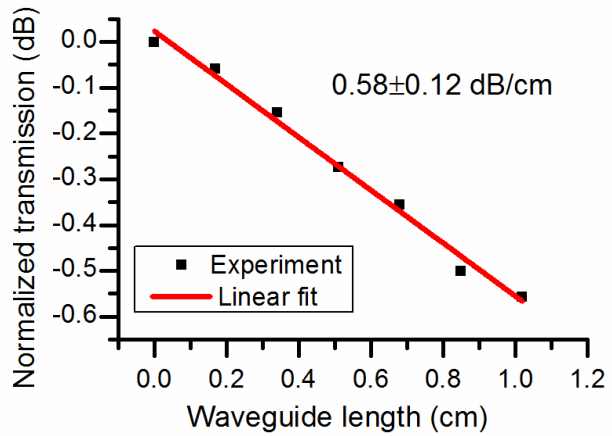

Fig. 6. Propagation loss measurement for the $3 \mu \mathrm{m}$ GOS waveguides [27]

We also designed $1 \times 2$ and $2 \times 2$ MMI splitters, which are very useful devices for a range of photonic circuits. The $1 \times 2$ MMI dimensions were $\mathrm{W}_{\mathrm{MMI}}=10 \mu \mathrm{m}, \mathrm{L}_{\mathrm{MMI}}=58.2 \mu \mathrm{m}, \mathrm{W}_{\text {taper }}$ $=4.75 \mu \mathrm{m}, \mathrm{L}_{\text {taper }}=30 \mu \mathrm{m}$, and center to center output port separation $(\mathrm{S})=4.75 \mu \mathrm{m}$. The $2 \times 2$ MMI had the same dimensions apart from the length of the multimode region which was $\mathrm{L}_{\mathrm{MMI}}=114 \mu \mathrm{m}$. The MMI loss was measured by plotting the transmission through different numbers of successively linked MMIs. The measured loss was $0.21 \pm 0.02$ $\mathrm{dB} / \mathrm{MMI}$ for $1 \times 2$ MMIs [27]. We have also realized MachZehnder interferometers and angled MMIs in this platform, as well as single and cascaded ring/racetrack resonators [30].

\section{LONG WAVELENGTH MEASUREMENTS}

To our knowledge, the longest wavelength at which GOS waveguides have been demonstrated to date is $5.8 \mu \mathrm{m}$ [11]. Therefore, here we investigate GOS waveguides operating at $\lambda=7.5 \mu \mathrm{m}$ for the first time.

For waveguide characterization at $7.5 \mu \mathrm{m}$ wavelength, light from a tunable quantum cascade laser (Pranalytica) was focused with a $\mathrm{ZnSe}$ objective lens into a single mode $\mathrm{As}_{2} \mathrm{Se}_{3}$ fibre with a core diameter of about $12 \mu \mathrm{m}$ (IRflex). The other end of the fibre was butt coupled into the waveguide end facet and the output was imaged from top using a long wave infrared (LWIR) camera (FLIR SC660) with a detection range from $7.5-13 \mu \mathrm{m}$ and with a thermal sensitivity of $<30 \mathrm{mK}$. Fig. 7 shows a schematic of the experimental setup.

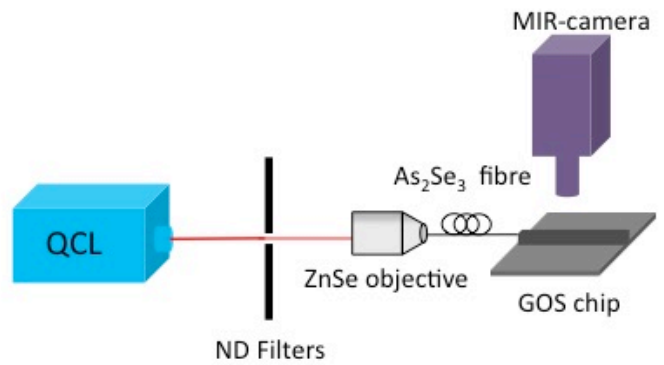

Fig. 7. Long wavelength measurement setup

In order to measure the waveguide propagation loss, we used the circuit shown in schematic form in Fig. 8, which consisted of a single waveguide that begins at a diced chip facet with a wide taper, then leads to a splitting tree which uses three stages of $1 \times 2$ MMIs as $-3 \mathrm{~dB}$ splitters to divide the light into 8 waveguides. Each of these 8 waveguides has a different length, and is terminated by a grating coupler that directs light up towards the LWIR camera. The relative transmissions of each of the waveguides can be used to calculate the waveguide loss, while the use of a single input waveguide and a splitting tree allows the measurement to be performed with just one alignment, by capturing one image of the surface of the chip when the laser is switched on, and one image for normalization in which the laser has been switched off.

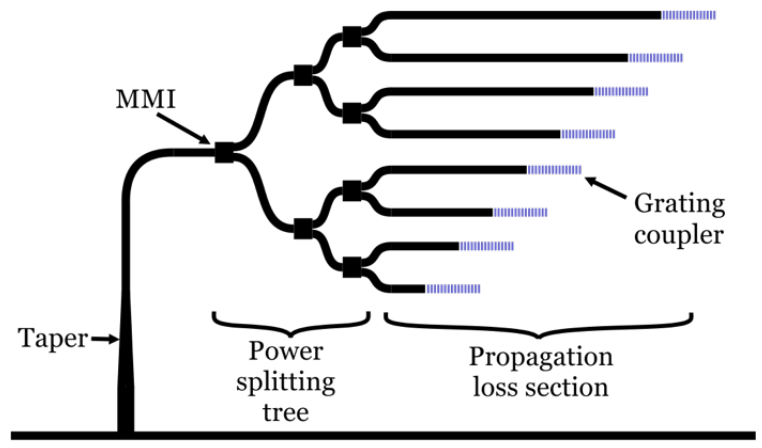

Diced facet

Fig. 8. Schematic diagram of the photonic circuit used to measure the GOS waveguide propagation loss at $\lambda=7.5 \mu \mathrm{m}$

We designed single mode GOS waveguides for this wavelength with the following dimensions: $\mathrm{H}=3.0 \mu \mathrm{m}, \mathrm{D}=$ $1.7 \mu \mathrm{m}$, and $\mathrm{W}=4.3 \mu \mathrm{m}$. The waveguide width at the facet is $35 \mu \mathrm{m}$, and a $1 \mathrm{~mm}$ long taper leads to the single mode waveguide. The bend radius was $300 \mu \mathrm{m}$ as simulations predicted it would give negligible bend loss. Each $1 \times 2 \mathrm{MMI}$ had tapered input and output ports $\left(\mathrm{L}_{\text {taper }}=100 \mu \mathrm{m}\right.$ and $\mathrm{W}_{\text {taper }}$ $=9.7 \mu \mathrm{m})$. The MMI multimode region was $91.4 \mu \mathrm{m}$ long and $20 \mu \mathrm{m}$ wide, and the MMI output ports had centre-to-centre spacing of $10.3 \mu \mathrm{m}$. S-bends connected the three stages of the splitter, having length $=1500 \mu \mathrm{m}, 750 \mu \mathrm{m}$, and $350 \mu \mathrm{m}$ and 
offset $=595 \mu \mathrm{m}, 295 \mu \mathrm{m}$, and $145 \mu \mathrm{m}$ in the 1st, 2nd and 3rd stage, respectively. The grating couplers had a period of 2.0 $\mu \mathrm{m}$ and duty cycle of 0.5 , and were $400 \mu \mathrm{m}$ long and $35 \mu \mathrm{m}$ wide, with an etch depth of $1.0 \mu \mathrm{m}$.

Fabrication was carried out using e-beam lithography and ICP etching, as described in section III. This time, two lithography and etching steps were used - the first to create the grating couplers, and the second for the other devices. The waveguide facet was prepared by dicing.

Fig. 9 shows the top view image of the waveguide loss section, taken using the LWIR camera when the laser is switched on. The background is then subtracted, leaving only the laser light. The transmission of each grating was calculated by summing the values of the pixels (which are proportional to the light intensity) in the region surrounding each grating coupler. The normalized relative transmission values for each waveguide length have been used to calculate the waveguide loss per unit length. The measured waveguide loss at $7.5 \mu \mathrm{m}$ was $3.0 \pm 0.8 \mathrm{~dB} / \mathrm{cm}$. This larger value of the propagation loss compared to the one in section III, is most likely due to higher mode interaction with both the Ge-Si interface region and the etched waveguide sidewalls.

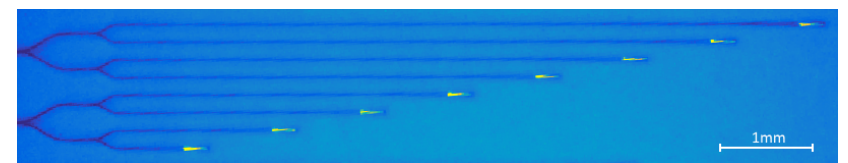

Fig. 9. Top view image of the waveguide loss section taken by a MIR camera.

\section{AMORPHOUS GERMANIUM}

While loss levels demonstrated in crystalline GOS [e.g. 27, 18] may not be achievable in amorphous germanium (a-Ge) [31], a-Ge can be deposited in a PECVD system, making it cheaper and more easily producible than the crystalline material, particularly on glass substrates. Amorphous Ge has been previously used as core material in optical fibers, where a high pressure chemical deposition method was followed, pumping a mixture of germane and helium through the core of a silica capillary, to achieve a minimum loss of $4.8 \mathrm{~dB} / \mathrm{cm}$ at $10.6 \mu \mathrm{m}$ wavelength [15]. Here we demonstrate, to our knowledge, the first MIR a-Ge planar waveguides.

Material thickness for the a-Ge was set at $500 \mathrm{~nm}$. Silicon dioxide was used as the bottom cladding, since the oxide material loss at the wavelength of $3.8 \mu \mathrm{m}$ is approximately 5 $\mathrm{dB} / \mathrm{cm}$ and should not result in large propagation losses, as shown in the SOI platform [e.g. 32-37]. Film mode matching simulations in Photon Design Fimmwave [26] revealed that a width of $1.3 \mu \mathrm{m}$ would be optimal to achieve minimum loss while retaining single mode operation.

An important aspect of this investigation was the use of Design of Experiments (DoE) to accurately capture the effect of deposition parameters with the least number of experimental runs. The first phase of using DoE is called screening and aims to select the most influential deposition parameters. Screening is followed by optimization were the effect of a small number (2-3) of important factors on the desired response is studied and analysed. The investigated parameters and their ranges are shown in Table I.

\begin{tabular}{ccccc}
\multicolumn{5}{c}{ TABLE I. DOE FOR A-GE SCREENING } \\
\hline \hline Run & $\mathrm{T}\left({ }^{\circ} \mathrm{C}\right)$ & $\mathrm{P}(\mathrm{W})$ & $\mathrm{H}_{2} / \mathrm{GeH}_{4}$ & $\mathrm{P}$ (mTorr) \\
\hline 1 & 200 & 25 & 5 & 500 \\
2 & 100 & 15 & 5 & 300 \\
3 & 200 & 15 & 5 & 500 \\
4 & 200 & 15 & 1 & 300 \\
5 & 200 & 25 & 1 & 300 \\
6 & 200 & 15 & 1 & 500 \\
7 & 100 & 25 & 1 & 500 \\
8 & 100 & 25 & 5 & 300
\end{tabular}

The $\mathrm{SiO}_{2}$ layer (4.6 $\mu$ m thick) was grown thermally at 1000 ${ }^{\circ} \mathrm{C}$. Prior to deposition, the samples were cleaned using acetone, IPA, and DI water. The a-Ge layers were deposited on the oxide layer using SYS100 PECVD system from Oxford Instruments. The devices were then patterned by e-beam lithography and were transferred to the $500 \mathrm{~nm}$ thick a-Ge using $\mathrm{SF}_{6} / \mathrm{C}_{4} \mathrm{~F}_{8}$ ICP etch. The average etch rate of the a-Ge was approximately $3.5 \mathrm{~nm} / \mathrm{s}$. Time dependent hazing has been observed in the fabricated devices, this appears as small accumulations of material on the surface. This is a known effect caused by the contact of Ge and moisture in air resulting in an oxidation processes. Further work needs to be carried out to investigate what coating materials can be used to isolate the surface of the a-Ge and to eliminate the oxidation process.

Grating couplers were used to couple light into $1.3 \mu \mathrm{m}$ wide a-Ge waveguides of different lengths. Minimum measured propagation loss was $6.9 \mathrm{~dB} / \mathrm{cm}$ (Fig. 10) for run 6 (Table I), which is significantly lower than $17 \mathrm{~dB} / \mathrm{cm}$ measured in the fibres at a similar wavelength [15]. Values for other fabrication runs varied between 7 and $20 \mathrm{~dB} / \mathrm{cm}$. Future work will include another DoE with fewer parameters, and a focus on optimizing the propagation loss. Moreover, the stress of the a-Ge layer will be studied, as will the use of cladding materials like aluminium oxide for avoiding degradation of the a-Ge surface while still retaining the Ge transparency range.

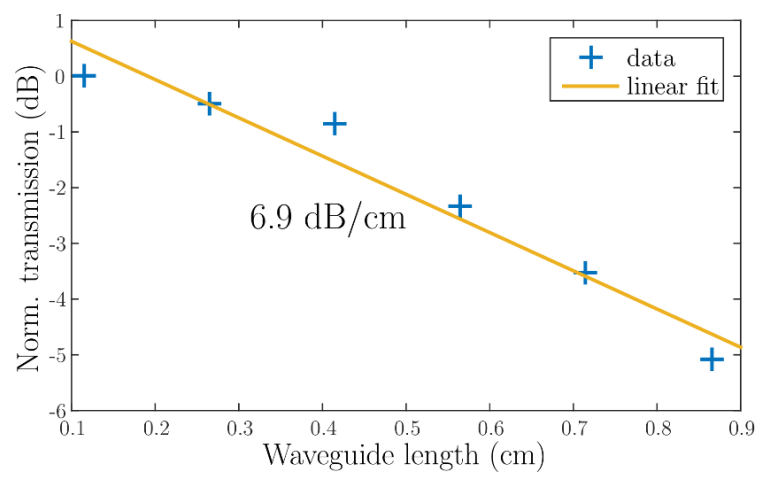

Fig. 10. Cut-back propagation loss measurements of a-Ge waveguides

\section{CONCLUSION}

Germanium is the most promising group IV material for a host of applications in the MIR wavelength range. It has significantly larger transparency window than $\mathrm{Si}$, as well as higher carrier mobility and nonlinear coefficients. If we add 
potential for the realization of active photonic devices (sources, modulators and detectors) in GeSn alloys, it is clear that Ge-based MIR photonic devices and systems can be applied in a range of applications, that include communications, clinical diagnostics, and astronomy.

In this paper, we have reviewed our recent results on Ge-onSi platform. We have achieved record low propagation loss of $0.6 \mathrm{~dB} / \mathrm{cm}$ at a wavelength of $3.8 \mu \mathrm{m}$, for $3 \mu \mathrm{m}$ high $\mathrm{Ge}$ waveguides. This significant reduction of propagation loss compared to the previously published data is a result of reduced interaction of the optical mode with threading dislocations at the Ge-Si interface and with sidewall roughness. We have also realized low loss MMI splitters (0.2$0.3 \mathrm{~dB} / \mathrm{MMI})$ as well as interferometers, spectrometers, and cascaded ring resonators in this platform. The two photon absorption coefficient in these waveguides has been measured and all-optical modulation demonstrated for the first time by using cross absorption modulation, achieving the extinction ratio of $8.1 \mathrm{~dB}$, the highest reported in any of the group IV waveguides to date. Following our measurements in the $2-$ $3.8 \mu \mathrm{m}$ wavelength range, we have conducted propagation loss measurements at $7.5 \mu \mathrm{m}$, and obtained a value of $\sim 3 \mathrm{~dB} / \mathrm{cm}$. This higher loss compared to the one at $3.8 \mu \mathrm{m}$, is a consequence of a larger interaction of the optical mode with dislocations and sidewall roughness. Finally, we have fabricated a-Ge waveguides and measured the propagation loss of $6.9 \mathrm{~dB} / \mathrm{cm}$ at $3.8 \mu \mathrm{m}$. Future work will include investigation of Ge-based platforms at longer wavelengths and their implementation in several application areas.

\section{REFERENCES}

[1] R. Soref, "Mid-infrared photonics in silicon and germanium," Nat Photon, vol. 4, pp. 495-497, 2010.

[2] D. J. Richardson, "Filling the light pipe," Science, vol. 330, pp. 327-328, 2010.

[3] T. Baehr-Jones, A. Spott, R. Ilic, A. Spott, B. Penkov, W. Asher, and M. Hochberg, "Silicon-on-sapphire integrated waveguides for the midinfrared," Opt. Express, vol. 18, pp. 12127-12135, 2010.

[4] F. Li, S. D. Jackson, C. Grillet, E. Magi, D. Hudson, S. J. Madden, Y. Moghe, C. O'Brien, A. Read, S. G. Duvall, P. Atanackovic, B. J. Eggleton, and D. J. Moss, "Low propagation loss silicon-on-sapphire waveguides for the mid-infrared," Opt. Express, vol. 19, pp. 15212$15220,2011$.

[5] R. Shankar, I. Bulu, and M. Lončar "Integrated high-quality factor silicon-on-sapphire ring resonators for the mid-infrared," Appl. Phys. Lett., vol. 102, 051108, 2013.

[6] S. Khan, J. Chiles, and S. Fathpour, "Silicon-on-nitride waveguides for mid- and near-infrared integrated photonics," Appl. Phys. Lett., vol. 102, 121104, 2013.

[7] G. Z. Mashanovich, M. M. Milosevic, M. Nedeljkovic, N. Owens, B. Xiong, E.-J. Teo, and Y. Hu, "Low loss silicon waveguides for the midinfrared," Opt. Express, vol. 19, pp. 7112-7119, 2011.

[8] Z. Cheng, X. Chen, C. Y. Wong, K. Xu, K. Y. Fung, Y. M. Chen, H. K. Tsang, "Focusing subwavelength grating coupler for mid-infrared suspended membrane waveguide," Opt. Lett., vol. 37, pp. 1217-1219, 2012.

[9] C. Reimer, M. Nedeljkovic, D. J. M. Stothard, M. O. S. Esnault, C. Reardon, L. O'Faolain, M. Dunn, G. Z. Mashanovich, and T. F. Krauss, "Mid-infrared photonic crystal waveguides in SOI," Opt. Express, vol. 20, pp. 29361-29368, 2012.

[10] J. Soler Penades, C. Alonso-Ramos, A. Z. Khokhar, M. Nedeljkovic, L. A. Boodhoo, A. Ortega-Monux, I. Molina-Fernandez, P. Cheben, and G. Z. Mashanovich, "Suspended SOI waveguide with sub-wavelength grating cladding for mid-infrared," Opt. Lett., vol. 39, pp. 5661-5664 (2014).
[11] Y.-C. Chang, V. Paeder, L. Hvozdara, J.-M. Hartmann, and H. P. Herzig, "Low-loss germanium strip waveguides on silicon for the midinfrared," Opt. Lett., vol. 37, pp. 2883-2885, 2012.

[12] A. Malik, M. Muneeb, S. Pathak, Y. Shimura, J. Van Campenhout, R. Loo, and G. Roelkens "Germanium-on-silicon mid-infrared arrayed waveguide grating multiplexers," IEEE Photon. Technol. Lett., vol. 25, pp. 1805-1808, 2013.

[13] G. Roelkens, U. Dave, A. Gassenq, N. Hattasan, C. Hu, B. Kuyken, F. Leo, A. Malik, M. Muneeb, E. Ryckeboer, Z. Hens, R. Baets, Y. Shimura, F. Gencarelli, B. Vincent, R. Loo, J. Van Campenhout, L. Cerutti, J.-B. Rodriguez, E. Tournié, X. Chen, M. Nedeljkovic, G. Z. Mashanovich, S. Li, N. Healy, A. C. Peacock, X. Liu, R. Osgood, and W. J. Green, "Silicon-based heterogeneous photonic integrated circuits for the mid-infrared," Opt. Mat. Express, vol. 3, pp. 1523-1536, 2013.

[14] A. Malik, S. Dwivedi, L. Van Landschoot, M. Muneeb, Y. Shimura, G. Lepage, J. Van Campenhout, W. Vanherle, T Van Opstal, R. Loo, and G. Roelkens, "Ge-on-Si and Ge-on-SOI thermo-optic phase shifters for the mid-infrared," Opt. Express, vol. 22, pp. 28479-28488, 2014.

[15] P. Mehta, M. Krishnamurthi, N. Healy, N. F. Baril, J. R. Sparks, P. J. A. Sazio, V. Gopalan, J. V. Badding, and A. C. Peacock, "Mid-infrared transmission properties of amorphous germanium optical fibers," Appl. Phys. Lett., vol. 97, 071117, 2010.

[16] S. E. Plunkett, S. Propst, and M. S. Braiman, "Supported planar germanium waveguides for infrared evanescent-wave sensing," Appl. Opt., vol. 36, pp. 4055-4061, 1997.

[17] L. Souriau, T. Atanasova, V. Terzieva, A. Moussa, M. Caymax, R. Loo, M. Meuris, and W. Vandervorst, "Characterization of threading dislocations in thin germanium layers by defect etching: toward chromium and HF-free solution," J. Electrochem. Soc., vol. 155, pp. H677-H681, 2008.

[18] L. Shen, N. Healy, C. J. Mitchell, J. Soler Penades, M. Nedeljkovic, G. Z. Mashanovich, and A. C. Peacock, "Mid-infrared all-optical modulation in low loss germanium-on-silicon waveguides," Opt. Lett., vol. 40, pp. 268-271, 2015.

[19] L. Shen, N. Healy, P. Mehta, T. D. Day, J. R. Sparks, J. V. Badding, and A. C. Peacock, "Nonlinear transmission properties of hydrogenated amorphous silicon core fibers towards the mid-infrared regime," Opt. Express, vol. 21, pp.13075, 2013.

[20] L. Shen, N. Healy, C. J. Mitchell, J. Solar Penades, M. Nedeljkovic, G. Z. Mashanovich, and A. C. Peacock, "Two-photon absorption and alloptical modulation in germanium-on-silicon waveguides for the midinfrared," Opt. Lett., vol. 40, pp. 2213-2216, 2015.

[21] B. V. Zubov, L. A. Kulevskii, V. P. Makarov, T. M. Murina and A. M. Prokhorov, "Two-photon absorption in germanium," JETP Letters, vol. 9, pp. 130, 1969.

[22] A. F. Gibson, C. B. Hatch, P. N. D. Maggs, D. R. Tilley, and A. C. Walker, "Two-photon absorption in indium antimonide and germanium," J. Phys. C, vol. 9, pp. 3259, 1976.

[23] X. Sang, E.-K. Tien, and O. Boyraz, "Applications of two-photon absorption in silicon," J. Optoelecton. Adv. M. ,vol.11, pp. 15, 2008.

[24] D. J. Moss, L. Fu, I. Littler, and B. J. Eggleton, "Ultrafast all-optical modulation via two-photon absorption in silicon-insulator waveguide," Electron. Lett., vol. 41, pp. 320, 2005.

[25] T. K. Liang, L. R. Nunes, T. Sakamoto, K. Sasagawa, T. Kawanishi, M. Tsuchiya, G. R. A. Priem, D. Van Thourhout, P. Dumon, R. Baets, and H. K. Tsang, "Ultrafast all-optical switching by cross-absorption modulation in silicon wire waveguides," Opt. Express, vol. 13, pp. 7298, 2005.

[26] Photon Design. [Online]. Available: http://www.photond.com/, accessed Jun. 30, 2016.

[27] M. Nedeljkovic, J. Soler Penades, C. J. Mitchell, T. Dominquez Bucio, A. Z. Khokhar, C. Littlejohns, F. Y. Gardes, and G. Z. Mashanovich, "Surface grating coupled low loss Ge-on-Si rib waveguides and multimode interferometers," IEEE Photon. Technol. Lett., vol. 27, pp. 1040-1043, 2015.

[28] Lumerical. [Online]. Available: https://www.lumerical.com/, accessed Jun. 30, 2016.

[29] C. Alonso-Ramos, M. Nedeljkovic, D. Benedikovic, J. Soler Penadés, C. Littlejohns, D. Pérez-Galacho, L. Vivien, P. Cheben, and G. Z. Mashanovich, "Germanium-on-silicon mid-infrared grating couplers with low-reflectivity inverse taper excitation," to be published

[30] B. Troia, J. Soler Penades, A. K. Khokhar, M. Nedeljkovic, C. AlonsoRamos, V. M. N. Passaro, and G. Z. Mashanovich, "Germanium-onsilicon Vernier-effect photonic microcavities for the mid-infrared," Opt. Lett., vol. 41, pp. 610-613, 2016. 
[31] T. E. Donovan and W. E. Spicer, "Optical properties of amorphous germanium films," Phys. Rev. B, vol. 2, pp. 397-413, 1970.

[32] J. Soler Penades, A. Z. Khokhar, M. Nedeljkovic, and G. Z. Mashanovich, "Low loss mid-infrared SOI slot waveguides," IEEE Photon. Technol. Lett., vol. 27, pp. 1197-1199, 2015.

[33] G. Z. Mashanovich, F. Y. Gardes, D. J. Thomson, Y. Hu, K. Li, M. Nedeljkovic, J. Soler Penades, A. Z. Khokhar, C. J. Mitchell, S. Stankovic, R. Topley, S. A. Reynolds, Y. Wang, B. Troia, V. M. N. Passaro, C. G. Littlejohns, T. Dominguez Bucio, P. R. Wilson, and G. T. Reed, "Silicon photonic waveguides and devices for near- and mid-IR applications," J. Sel. Top. Quantum Electron., vol. 21, 8200112, 2015.

[34] B. Troia, A. Z. Khokhar, M. Nedeljkovic, J. Soler Penades, V. M. N. Passaro, and G. Z. Mashanovich, "Cascade-coupled racetrack resonators based on the Vernier effect in mid-infrared," Opt. Express, vol. 22, pp. 23990-24003, 2014.
[35] M. Nedeljkovic, S. Stanković, C. Mitchell, A. Z. Khokhar, S. Reynolds, D. J. Thomson, F. Y. Gardes, C. Littlejohns, G. T. Reed, and G. Z. Mashanovich, "Mid-infrared thermo-optic modulators in SOI," IEEE Photon. Technol. Lett., vol. 26, pp. 1352-1355, 2014.

[36] Y. Hu, T. Li, D. J. Thomson, X. Chen, J. Soler Penades, A. Z. Khokhar, C. J. Mitchell, G. T. Reed, and G. Z. Mashanovich, "Wavelength division (de)multiplexing in mid-infrared wavelength range using interleaved angled multimode interferometer on the silicon-on-insulator platform," Opt. Lett., vol. 39, pp. 1406-1409, 2014.

[37] M. Nedeljkovic, A. Z. Khokhar, Y. Hu, X. Chen, J. Soler Penades, S. Stankovic, D. J. Thomson, F. Y. Gardes, H. M. H. Chong, G. T. Reed, and G. Z. Mashanovich, "Silicon photonic devices and platforms for the mid-infrared" Opt. Mat. Express, vol. 3, pp. 1205-1214, 2013. 